\title{
Telomerase expression in sebaceous lesions of the skin
}

\author{
Background: Recent studies have demonstrated telomerase expression \\ in ophthalmologic sebaceous carcinoma and have suggested possible \\ diagnostic utility in distinguishing these neoplasms from sebaceous \\ adenomas. The aim of this study was to evaluate telomerase expression \\ via human telomerase reverse transcriptase (hTERT) immunohisto- \\ chemical staining in a spectrum of sebaceous lesions of the skin. \\ Methods: Paraffin-embedded sections from sebaceous hyperplasia \\ (11), nevus sebaceus (22), sebaceous adenoma (19), sebaceoma (11), and \\ sebaceous carcinoma (14) were evaluated for intensity (0 to $3+)$ and \\ pattern of anti-hTERT staining. \\ Results: Strong (2 to $3+$ ) hTERT staining was observed in nucleoli of \\ germinative cells and immature sebocytes in all sebaceous lesions, \\ whereas mature sebocytes were negative. The distribution pattern \\ paralleled features seen by routine haematoxylin and eosin-stained \\ sections. \\ Conclusions: All hyperplastic and neoplastic sebaceous skin lesions \\ expressed hTERT in this immunohistochemical study. The pattern of \\ staining was predictive of the histologic pattern of the process but does \\ not significantly add to our diagnostic armamentarium of sebaceous \\ lesions. \\ Olsen SH, Su LD, Thomas D, Fullen DR. Telomerase expression in \\ sebaceous lesions of the skin. \\ J Gutan Pathol 2007; 34: 386-391. (c) Blackwell Munksgaard 2006.
}

Stephen H. Olsen ${ }^{1}$, Lyndon

D. Su ${ }^{1,2}$, Dafydd Thomas ${ }^{1,3}$ and Douglas R. Fullen ${ }^{1,2}$

${ }^{1}$ Department of Pathology,

${ }^{2}$ Department of Dermatology, and

${ }^{3}$ Department of Internal Medicine, University of Michigan Medical Center, Ann Arbor, MI, USA

Douglas R. Fullen, MD, M3261, Medical Sciences I, Department of Pathology, University of Michigan Medical Center, 1301 Catherine, Ann Arbor, Ml 48109-0602, USA

Tel: +1 7347644460

E-mail: dfullen@med.umich.edu

Accepted for publication May 15, 2006
Telomeres are tandem nucleotide repeats, (TTAG$\mathrm{GG})_{\mathrm{n}}$, located at the ends of chromosomes in all vertebrates; they function to protect chromosomes from degradation and aberrant recombination during replication. ${ }^{1-5}$ Telomere length or number of repeats, in turn, is maintained by the ribonucleoprotein DNA polymerase complex, telomerase, although alternate pathways exist. ${ }^{6,7}$ This complex is composed of an RNA moiety (hTR) and an RNAdependant DNA polymerase (hTERT). Telomerase expression is repressed in most human somatic cells, resulting in progressive loss of telomeres and shortening of chromosomes with successive cell divisions. Eventually, chromosomes reach a critical length at which cell division ceases, senescence begins, and the cells ultimately undergo apoptosis or cell death., In addition, telomerase activity has been detected in germline cells, somatic cells during fetal development, germinal centres of lymph nodes, regenerative epithelium of the gastrointestinal tract, proliferative endometrium, the bulge region of hair follicles, stem cells of the epidermis, sweat glands, and sebaceous glands. ${ }^{10-17}$

Immortalized cell lines of a vast array of human cancers, such as breast, colorectal and ovarian cancers, and melanoma, have been shown to overexpress telomerase. ${ }^{18-24} \mathrm{In}$ addition to melanoma, several other cutaneous neoplasms have been shown to express telomerase, including basal cell carcinoma, in situ and invasive squamous cell carcinoma, eccrine carcinoma, poroma and porocarcinoma, trichilemmal carcinoma, common acquired and Spitz nevi, and seborrheic keratosis. 16,17,25-27 Thus, telomerase is reputed to play a role in the unregulated proliferation and ultimate immortality of somatic cells in a variety of benign and malignant tumors. ${ }^{28-32}$

Telomerase has been identified in sebaceous carcinoma of the orbit, but not in sebaceous adenomas, 


\section{hTERT in sebaceous lesions}

by using both Telomerase Repeat Amplification Protocol (TRAP) and in situ hybridization (ISH) assays. Thus, a possible diagnostic role for telomerase in sebaceous lesions is suggested. ${ }^{33,34}$ Previous studies have reported telomerase expression in sebaceous glands of normal skin. However, telomerase expression has not been evaluated in extra-ocular sebaceous lesions to date. ${ }^{16,17}$ The goal of this study was to evaluate immunohistochemical staining for human telomerase reverse transcriptase (hTERT) expression in paraffin-embedded sections of a spectrum of hyperplastic and neoplastic sebaceous lesions of the skin to determine whether this method aids in differentiation among these lesions and to compare our results to those reported for ophthalmologic sebaceous tumors.

\section{Materials and methods}

Formalin-fixed, paraffin-embedded archival tissue blocks from 11 sebaceous hyperplasias, 22 cases of nevus sebaceus, 19 sebaceous adenomas, 11 sebaceomas, and 14 sebaceous carcinomas were retrieved following a SNOMED search of our laboratory data system. The diagnoses were rendered by one of three staff dermatopathologists on the Dermatopathology Service at the University of Michigan. Four cases (two sebaceous adenomas, one sebaceoma, and one sebaceous carcinoma) were diagnosed in patients known to have the Muir-Torre syndrome.

Immunohistochemical staining for hTERT was performed on all 77 sebaceous lesions. Briefly, 4- $\mu \mathrm{m}$ thick sections were cut and placed on charged slides. The slides were deparaffinized, rehydrated, and endogenous peroxidases were blocked by incubation with $3 \% \mathrm{H}_{2} \mathrm{O}_{2}$. Antigen retrieval was accomplished by incubating the slides with $10-\mathrm{mm}$ citrate buffer at pH 6.0 and microwaving for $20 \mathrm{~min}$. The slides were stained with a primary monoclonal antibody to hTERT (1:50; Novocastra, Newcastle, UK). The primary antibody titer used in this study was selected based on the dilution that produced an optimum staining pattern as judged in titration studies performed on tonsil and melanoma tissue microarray controls and was in concordance with the manufacturer's recommendation. Immunohistochemical staining was performed on a DAKO Autostainer (DAKO, Carpinteria, CA, USA) using DAKO LSAB + and 3,3-diaminobenzidine as the chromogen. The slides were counterstained with Mayer's haematoxylin. Positive and negative control slides were included. The staining intensity was scored semiquantitatively (negative/0, weak/1+, moderate/2+, strong/3+) with attention to cellular distribution and overall pattern of staining by all three observers, including two board certified dermatopathologists (LDS and DRF), with excellent concordance.

The Institutional Review Board of the University of Michigan has exempted this study from further review.

\section{Results}

\section{Demographics}

The 11 sebaceous hyperplasias came from the face of patients ranging from 27 to 89 years of age (mean 58, median 51). Nineteen sebaceous adenomas came from 18 patients ranging from 54 to 89 years of age (mean 70, median 71). With the exception of two cases (shoulder, chest), the sebaceous adenomas were located on the face. Eleven sebaceomas from 10 patients, 30-79 years of age (mean 57, median 55), were predominantly observed on the face, with the exception of three cases (shoulder, 1; back, 2). Fourteen sebaceous carcinomas arose in patients ranging from 49 to 92 years of age (mean 71, median 73) and were primarily located on the face, with the exception of three cases (chest, 1; back, 2). Twenty-one samples of nevus sebaceus came from the head and neck (primarily the scalp) of 20 patients ranging in age from 1 to 61 years of age (mean 17, median 15). All samples were from extraocular locations.

Many of the patients with sebaceous neoplasms had comorbid cutaneous neoplasms (basal cell carcinoma or squamous cell carcinoma). Seven patients had comorbid visceral malignancies (lung, colon, ovary, endometrium, and urinary bladder). Of these seven patients, four had undergone formal genetic counseling and testing to document hereditary non-polyposis colorectal cancer (HNPCG)associated abnormalities and were found to have the Muir-Torre syndrome. One sebaceous adenoma and one sebaceoma were diagnosed in patients with MLH-1 deletions, whereas one sebaceous adenoma and one sebaceous carcinoma were identified in patients with MSH-2 mutations.

\section{Staining}

All 77 sebaceous lesions showed some degree of positive staining for hTERT, with localization of staining in germinative cells and immature sebocytes across the spectrum of lesions in this study. The vast majority of positive cells displayed intense dot-like nucleolar staining (2 to $3+$ ); nuclear and cytoplasmic positivity was also noted, however, it was focal and generally less intense. Mature sebocytes were consistently negative. This distribution of staining imparted a pattern parallel with and predictive of 


\section{Olsen et al.}

the morphology seen by routine hematoxylin and eosin-stained sections.

The hTERT-positive cells observed in sebaceous hyperplasia and nevus sebaceus lesions were limited to the outer rim of the sebaceous lobules, corresponding to the germinative cell and immature sebocyte layers (Fig. 1). Sebaceous adenomas contained a greater number of more densely spaced positive cells because of staining of expanded layers of germinative and immature sebaceous cells. The hTERT staining highlighted the orderly growth of sebocytes and maintenance of a normal lobular polarity in these lesions (Fig. 2).

Sebaceomas and sebaceous carcinomas contained a higher percentage of hTERT-positive cells when compared with sebaceous adenomas. However, staining was distributed more diffusely throughout sebaceous lobules, highlighting an increase in architectural disorder of the lobules and loss of lobular polarity. This random distribution of hTERT was
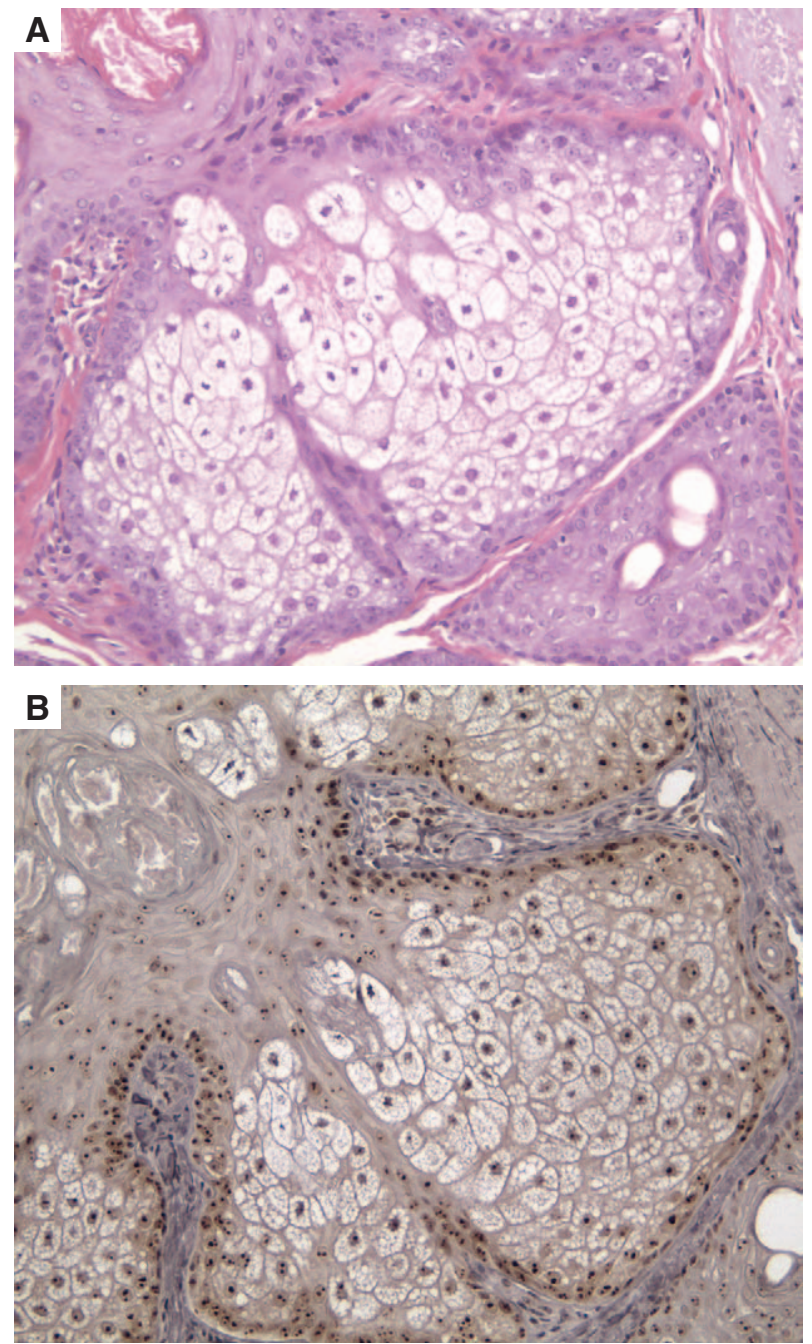

Fig. 1. Sebaceous hyperplasia. Human telomerase reverse transcriptase (hTERT)-positive cells are limited to the outer rim of the sebaceous lobule. (A) H\&E; (B) hTERT (×200).
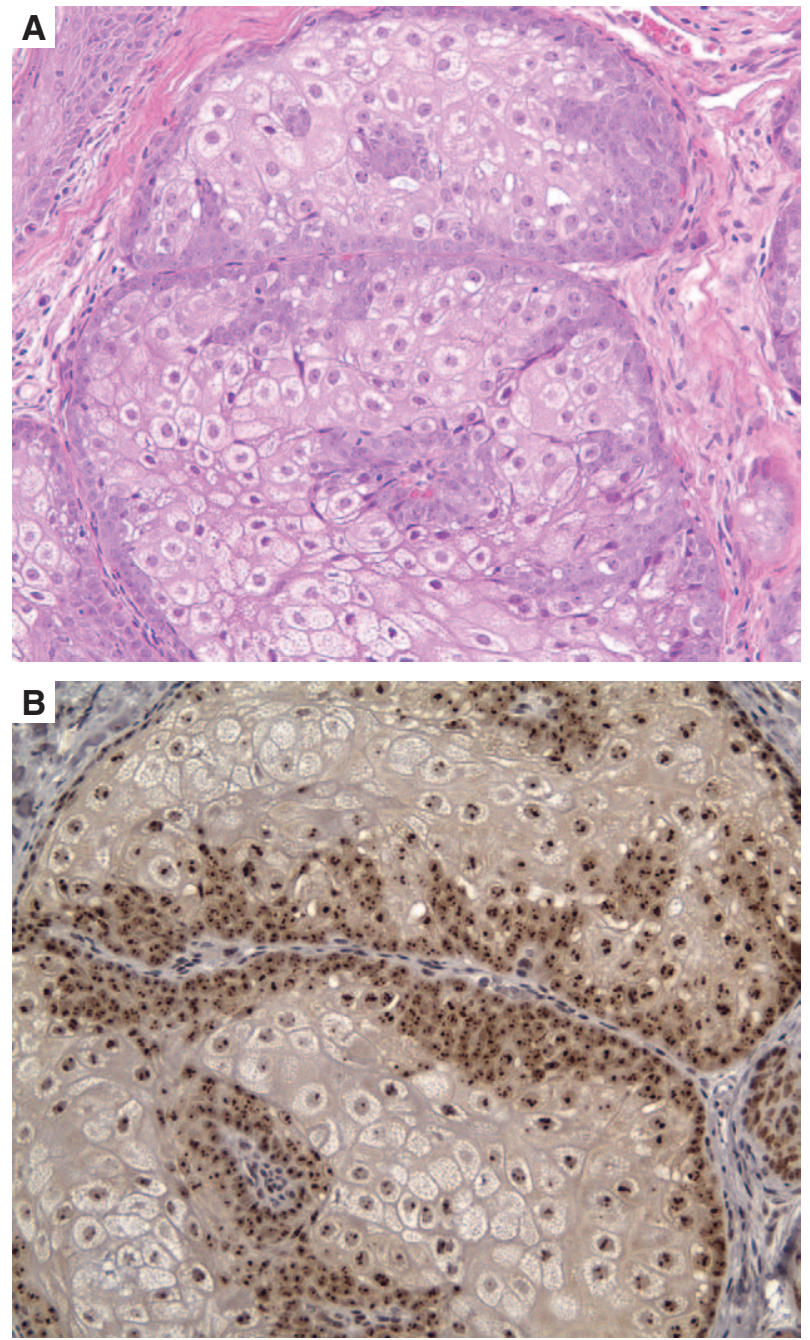

Fig. 2. Sebaceous adenoma. Increased number of positively staining cells, displaying ordered growth and appropriate lobular polarity. (A) H\&E; (B) hTERT $(\times 200)$.

similar in both types of lesions, such that they could not be distinguished based on hTERT staining alone (Figs 3 and 4).

There were no differences in morphology or telomerase staining intensity or distribution between patients with the Muir-Torre syndrome and the remainder of the study set.

A similar nucleolar pattern of hTERT staining was detected in keratinocytes of the epidermis and follicular epithelium, normal sebaceous lobules, and sweat glands. This staining pattern is consistent with previous observations of hTERT staining in normal skin. ${ }^{16,17}$

\section{Discussion}

This study confirmed that hTERT can be reliably detected in paraffin-embedded sections by immunoperoxidase staining. In fact, all sebaceous lesions 


\section{hTERT in sebaceous lesions}
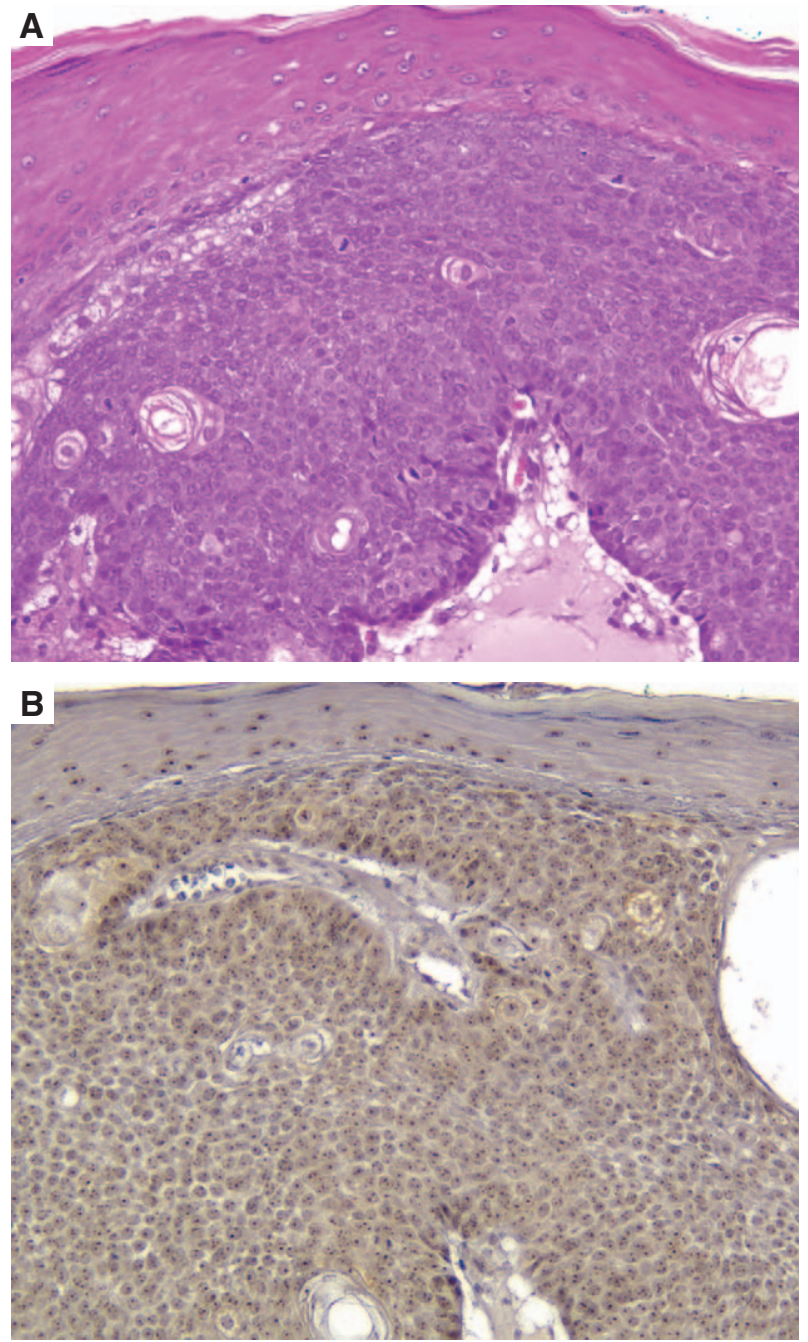

Fig. 3. Sebaceoma. Human telomerase reverse transcriptase (hTERT)-positive cells distributed diffusely, without lobular polarity. This is indistinguishable from the staining pattern of sebaceous carcinoma. (A) H\&E; (B) hTERT $(\times 200)$.

stained, to some extent, in an intense (2 to $3+$ ) nucleolar pattern. As previously noted, we also identified immuno-positivity in normal structures, such as normal sebaceous lobules and sweat glands, hair follicle epithelium, and keratinocytes at all levels of the epidermis. ${ }^{16,17}$ However, the staining seen in these normal structures was generally less intense, and more punctate or dot-like, relative to that of the neoplastic lesions.

Among the sebaceous hyperplasias and neoplasms, hTERT staining localized to the germinative cells and immature sebocytes. The staining intensity in these cell types was fairly uniform across the spectrum of lesions examined, regardless of the degree of differentiation. The differences noted between sebaceous lesions were in the distribution of the positively staining cells and not in the staining per se. As such, no quantitative differences between lesions were discerned.
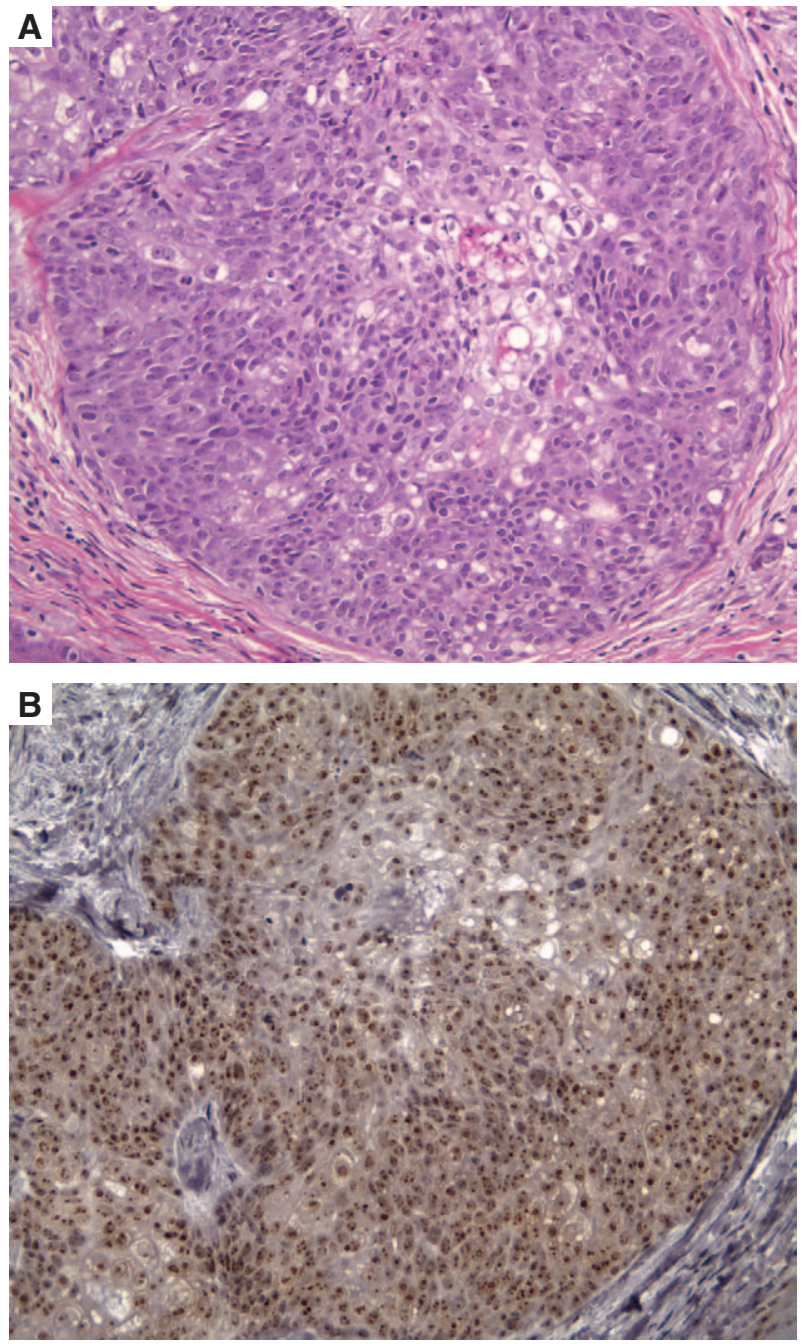

Fig. 4. Sebaceous carcinoma. Human telomerase reverse transcriptase (hTERT)-positive cells distributed diffusely without lobular polarity. This is indistinguishable from the staining pattern of sebaceoma. (A) H\&E; (B) hTERT $(\times 200)$.

On the basis of density and distribution of hTERT-positive cells, three groups of lesions could be identified. The first group, comprising sebaceous hyperplasia and nevus sebaceus lesions, contained a low density of positive cells that were regularly spaced and limited to the outer rim of sebaceous lobules. The second group had greater numbers of positive cells, displaying orderly growth and appropriate polarity within lobules, corresponding to sebaceous adenomas. The last group had the greatest number of positive cells, which were diffusely distributed throughout lobules in a disorganized fashion, with loss of the normal lobular polarity; this group consisted of sebaceomas and sebaceous carcinomas. We could not reliably distinguish sebaceomas from sebaceous carcinomas on the basis of hTERT staining intensity or pattern. Overall, the hTERT staining results correlated well with the diagnosis rendered on routine histologic 


\section{Olsen et al.}

examination and did not provide additional diagnostic information.

In recent studies, $\mathrm{Li}$ et al. studied a series (55 cases) of sebaceous carcinomas by TRAP and then ISH for hTR and hTERT and found them to be positive by ISH in 85 and $58 \%$ of their cases, respectively. In contrast, four sebaceous adenomas and adjacent uninvolved skin in their study were negative, suggesting a possible diagnostic role for telomerase ISH in the evaluation of ocular sebaceous neoplasms. These findings differ from the uniform strong hTERT staining across the spectrum of sebaceous lesions seen in our study. The reason for this difference is not entirely clear but may reflect differences in the methodologies used. Of their positive cases, many displayed only weak or moderate intensity $(<50 \%$ of 'signal area'). This could represent lower sensitivity of their ISH method because of weak indicator signal level or possibly loss of nucleic acid in their archival paraffin-embedded tissue. $\mathrm{Li}$ et al. also reported an inverse relationship between hTR and hTERT levels and the degree of morphologic differentiation. Although we did not note difference relative to the degree of differentiation, this is at least superficially similar to our finding of hTERT localized and distributed in the more immature cells of the lesions.

There was no difference in telomerase staining in the cases from patients with the Muir-Torre syndrome compared with sporadic sebaceous neoplasms. However, we cannot exclude the possibility that some of our cases considered to be sporadic were not early manifestations of the Muir-Torre syndrome, because not all the cases were evaluated for mutations in mismatch repair genes. The MuirTorre syndrome and HNPCG genotype/phenotype imply a predisposition to carcinogenesis through mutations in the DNA mismatch repair system or alternate pathways, as recently described by Goldberg et al. ${ }^{35}$ Intuitively, this is upstream in the pathway. Although up-regulation of telomerase is required for ultimate immortalization of cells, its role in carcinogenesis is unclear. These data suggest that the genetic alterations seen in HNPCG are independent of telomerase expression. Although it appears that telomerase expression cannot be used as a surrogate marker for the Muir-Torre syndrome, the number of patients with documentation of this condition was low in this study and, therefore, limits the interpretation of the data in this subset of patients.

In summary, hTERT expression was found in hyperplastic, hamartomatous, and neoplastic sebaceous lesions of the skin, as well as many normal skin structures. Staining intensity was uniformly strong, predominantly in a nucleolar pattern. Reproducible hTERT staining of germinative and immature sebocytes resulted in separation of extraocular sebaceous lesions into three groups: sebaceous hyperplasia and nevus sebaceus; sebaceous adenoma; and sebaceoma and sebaceous carcinoma. The overall staining pattern for hTERT confirmed the diagnosis rendered on routine histologic examination and does not seem to provide additional diagnostic information. Additionally, no difference was noted in the cases of the Muir-Torre syndrome. As such, hTERT offered no apparent diagnostic utility for sebaceous neoplasms.

\section{Acknowledgements}

These findings were presented in abstract form at the 2005 Annual Meeting of the American Society of Dermatopathology. Dr Dafydd Thomas is a Walther Cancer Research Institute Scholar and thanks them for their support.

\section{References}

1. Blackburn EH. Structure and function of telomeres. Nature 1991; 350: 569.

2. Greider CW. Mammalian telomere dynamics: healing, fragmentation shortening and stabilization. Curr Opin Genet Dev 1994; 4: 203.

3. Harley CB, Futcher AB, Greider GW. Telomeres shorten during ageing of human fibroblasts. Nature 1990; 345: 458.

4. Allsopp RC, Vaziri H, Patterson C, et al. Telomere length predicts replicative capacity of human fibroblasts. Proc Natl Acad Sci USA 1992; 89: 10114.

5. Blackburn EH. Telomeres: structure and synthesis. J Biol Chem 1990; 265: 5919.

6. Blackburn EH, Greider CW, Henderson E, Lee MS, Shampay J, Shippen-Lentz D. Recognition and elongation of telomeres by telomerase. Genome 1989; 31: 553.

7. Blackburn EH. Telomerases. Annu Rev Biochem 1992; 61: 113.

8. Olovnikov AM. A theory of marginotomy. The incomplete copying of template margin in enzymic synthesis of polynucleotides and biological significance of the phenomenon. $J$ Theor Biol 1973; 41: 181.

9. Allsopp RC, Harley CB. Evidence for a critical telomere length in senescent human fibroblasts. Exp Cell Res 1995; 219: 130.

10. Wright WE, Piatyszek MA, Rainey WE, Byrd W, Shay JW. Telomerase activity in human germline and embryonic tissues and cells. Dev Genet 1996; 18: 173.

11. Bonatz G, Klapper W, Barthe A, et al. Analysis of telomerase expression and proliferative activity in the different layers of cyclic endometrium. Biochem Biophys Res Commun 1998; 253: 214.

12. Yasumoto S, Kunimura C, Kikuchi K, et al. Telomerase activity in normal human epithelial cells. Oncogene 1996; 13: 433.

13. Brien TP, Kallakury BV, Lowry CV, et al. Telomerase activity in benign endometrium and endometrial carcinoma. Cancer Res 1997; 57: 2760.

14. Ramirez RD, Wright WE, Shay JW, Taylor RS. Telomerase activity concentrates in mitotically active segments of human hair follicles. J Invest Dermatol 1997; 108: 113.

15. Harle-Bachor C, Boukamp P. Telomerase activity in the regenerative basal layer of the epidermis in human skin and in 


\section{hTERT in sebaceous lesions}

immortal and carcinoma-derived skin keratinocytes. Proc Natl Acad Sci USA 1996; 93: 6476.

16. Guttman-Yassky E, Bergman R, Manov L, Sprecher E, Shaefer Y, Kerner H. Human telomerase RNA component expression in Spitz nevi, common melanocytic nevi, and malignant melanomas. J Cutan Pathol 2002; 29: 341.

17. Fullen D, Zhu W, Thomas D, Su L. hTERT expression in melanocytic lesions: an immunohistochemical study on paraffin-embedded tissue. J Cutan Pathol 2005; 32: 680.

18. Hiyama E, Gollahon L, Kataoka T, et al. Telomerase activity in human breast tumors. J Natl Cancer Inst 1996; 88: 116.

19. Landberg G, Nielsen NH, Nilsson P, et al. Telomerase activity is associated with cell cycle deregulation in human breast cancer. Cancer Res 1997; 57: 549.

20. Yashima K, Milchgrub S, Gollahon L S, et al. Telomerase enzyme activity and RNA expression during the multistage pathogenesis of breast carcinoma. Clin Cancer Res 1998; 4: 229.

21. Counter CM, Hirte HW, Bacchetti S, Harley CB. Telomerase activity in human ovarian carcinoma. Proc Natl Acad Sci USA 1994; 91: 2900 .

22. Duggan BD, Wan M, Yu MC, et al. Detection of ovarian cancer cells: comparison of a telomerase assay and cytologic examination. J Natl Cancer Inst 1998; 90: 238.

23. Tahara H, Kuniyasu H, Yokozaki H, et al. Telomerase activity in preneoplastic and neoplastic gastric and colorectal lesions. Clin Cancer Res 1995; 1: 1245.

24. Tang R, Cheng AJ, Wang JY, Wang TC. Close correlation between telomerase expression and adenomatous polyp progression in multistep colorectal carcinogenesis. Cancer Res 1998; 58: 4052.

25. Taylor RS, Ramirez RD, Ogoshi M, Chaffins M, Piatyszek MA, Shay JW. Detection of telomerase activity in malignant and non-malignant skin conditions. J Invest Dermatol 1996; 106: 759.

26. Parris CN, Jezzard S, Silver A, et al. Telomerase activity in melanoma and non-melanoma skin cancer. Br J Cancer 1999; 79: 47.

27. Wu A, Ichihashi M, Ueda M. Correlation of the expression of human telomerase subunits with telomerase activity in normal skin and skin tumors. Cancer 1999; 86: 2038.

28. Counter CM, Avilion AA, LeFeuvre CE, et al. Telomere shortening associated with chromosome instability is arrested in immortal cells which express telomerase activity. EMBO J 1992; 11: 1921.

29. Holt SE, Wright WE, Shay JW. Regulation of telomerase activity in immortalized cell lines. Mol Cell Biol 1996; 16: 2932.

30. Shay JW. Telomerase in human development and cancer. J Cell Physiol 1997; 173: 266.

31. Kim NW, Piatyszek MA, Prowse KR, et al. Specific association of human telomerase activity with immortal cells and cancer. Science 1994; 266: 2011.

32. Shay JW, Bacchetti S. A survey of telomerase activity in human cancer. Eur J Cancer 1997; 33: 787.

33. Li B, Li N, Xu X, et al. Telomerase expression in sebaceous carcinoma of the eyelid. Chin Med J 2004; 117: 445.

34. Li B, Li N, Guangyin C, et al. Correlation of the expression of telomerase RNA with the risk factors for recurrence of sebaceous gland carcinoma. Graefe's Arch Clin Exp Ophthalmol 2006; 244: 480.

35. Goldberg M, Rummelt C, Foja S, Holbach LM, Ballhausen WG. Different genetic pathways in the development of periocular sebaceous gland carcinomas in presumptive Muir-Torre Syndrome patients. Hum Mutat 2006; 27: 155. 